\title{
A web application for follow-up of results from a mobile device test battery for Parkinson's disease patients
}

Mevludin Memedi ${ }^{1}$, Jerker Westin ${ }^{1,2}$, Dag Nyholm ${ }^{3}$, Mark Dougherty ${ }^{1}$, Torgny Groth ${ }^{2}$

${ }^{1}$ Academy of Industry and Society, Computer Science, Dalarna University, Borlänge;

Medical Sciences, Biomedical Informatics and Engineering, Uppsala University, Uppsala; ${ }^{3}$ Neuroscience, Neurology, Uppsala University, Uppsala, Sweden

\section{Background}

A test battery consisting of self-assessments and motor tests (tapping and spiral drawing) was developed for a hand computer with touch screen in a telemedicine setting.

\section{Objective}

To develop and evaluate a web-based system that delivers decision support information to the treating clinical staff for assessing Parkinson's disease (PD) symptoms in their patients based on the test battery data.

\section{Methods}

The test battery is currently being used in a clinical trial (DAPHNE, EudraCT No. 2005-002654-21) by sixtyfive patients with advanced PD on 9991 test occasions (four tests per day during 362 week-long test periods) at nine clinics around Sweden [1-4].

Test results are sent continuously from the hand unit over a mobile net to a central computer and processed with statistical methods (fig 1). They are summarized into scores for different dimensions of the symptom state and an "overall test score" reflecting the overall condition of the patient during a test period. The system architecture consists of different modules pipelined to perform the main tasks, such as data collection, processing and presentation (fig 2).

The information in the web application is organized and presented graphically in a way that the general overview of the patient performance per test period is emphasized. Focus is on the overall test score, symptom dimensions and daily summaries. Dimensions of the symptom severity are illustrated in the form of a regular hexagon (fig 3). In addition to this, in another page a table of statistical summaries of the patient and reference group is shown. An example taken from the web application is shown (fig 4).

In a recent preliminary user evaluation, the web application was demonstrated to the fifteen study nurses who had used the test battery in the clinical trial where at least one patient per clinic was shown.

\section{Figure 1.}

System description. The patient answers questions and performs tests on several occasions per day during test periods of about one week. Data is transmitted over the mobile net to a central server for processing. The web application graphically presents the feedback on test results to the treating clinical staff to assist in decision making concerning treatment.

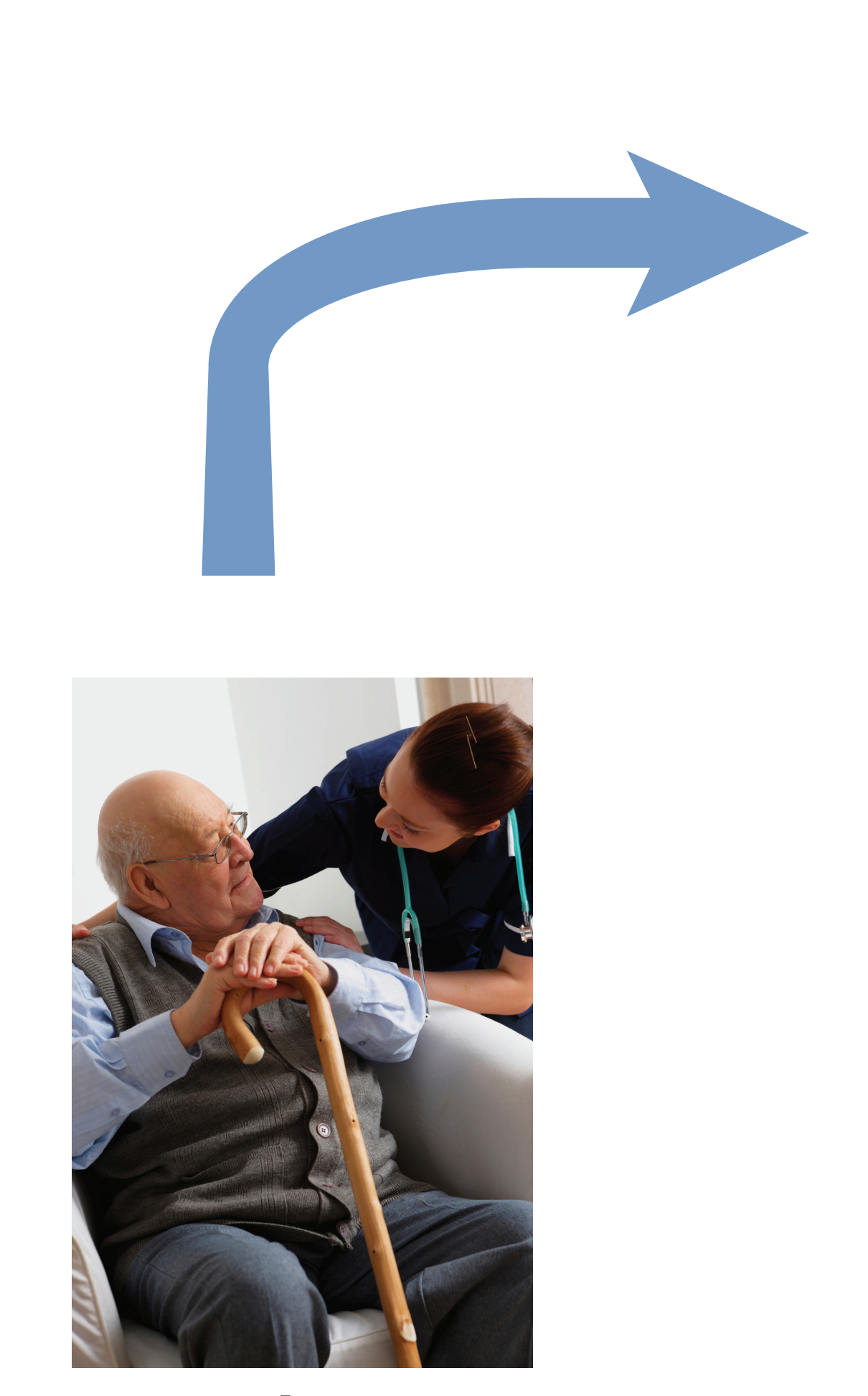

Parkinson Patient

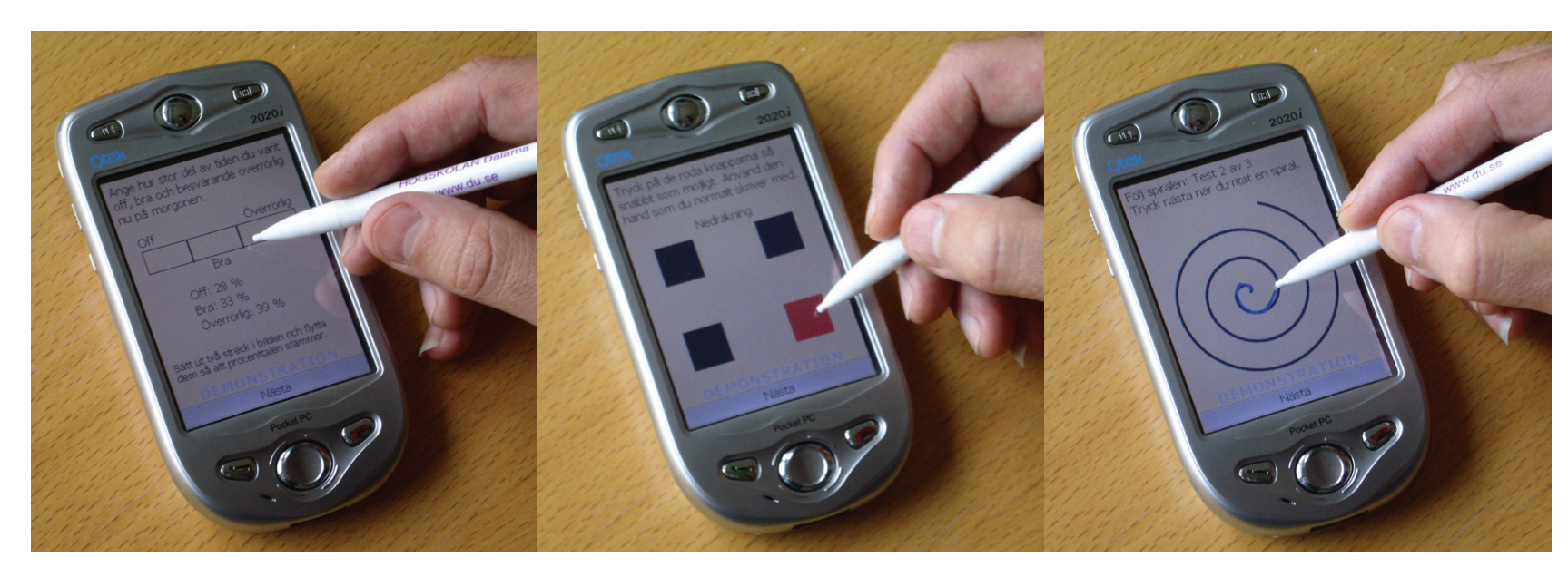

- Test Battery Diary questions - Motor tests

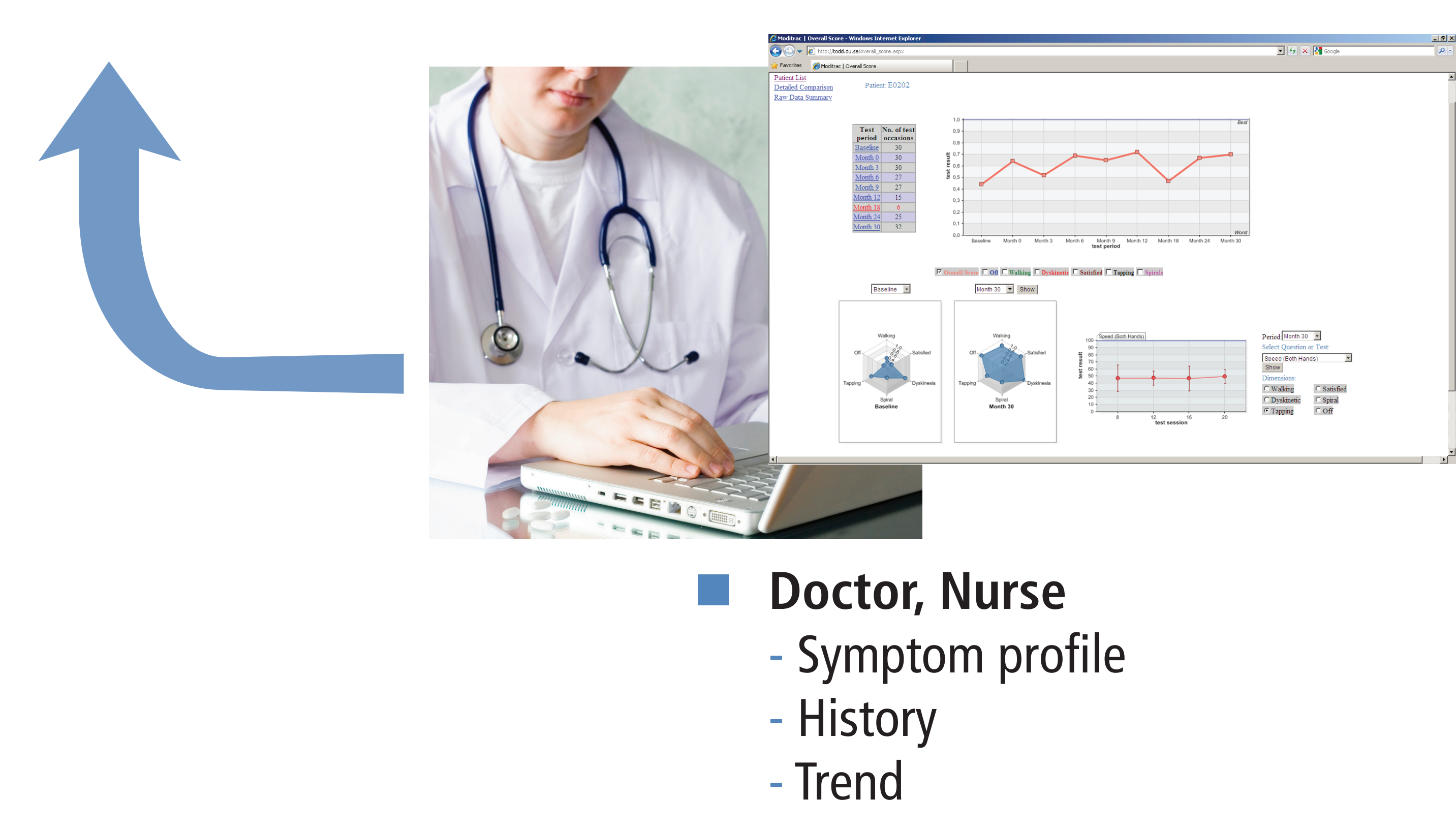

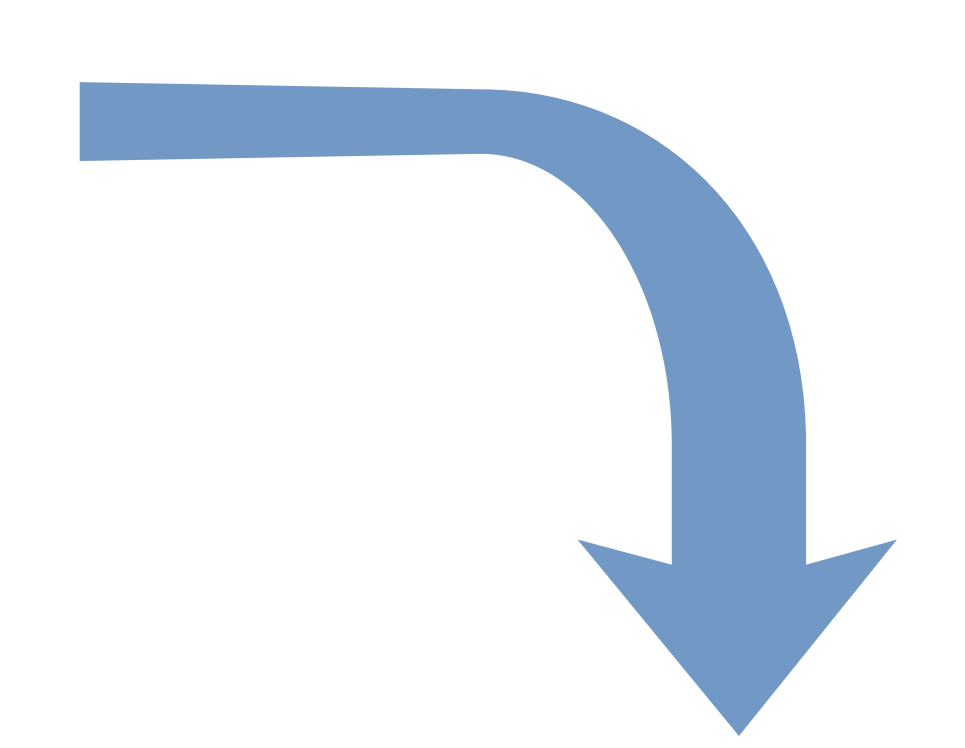

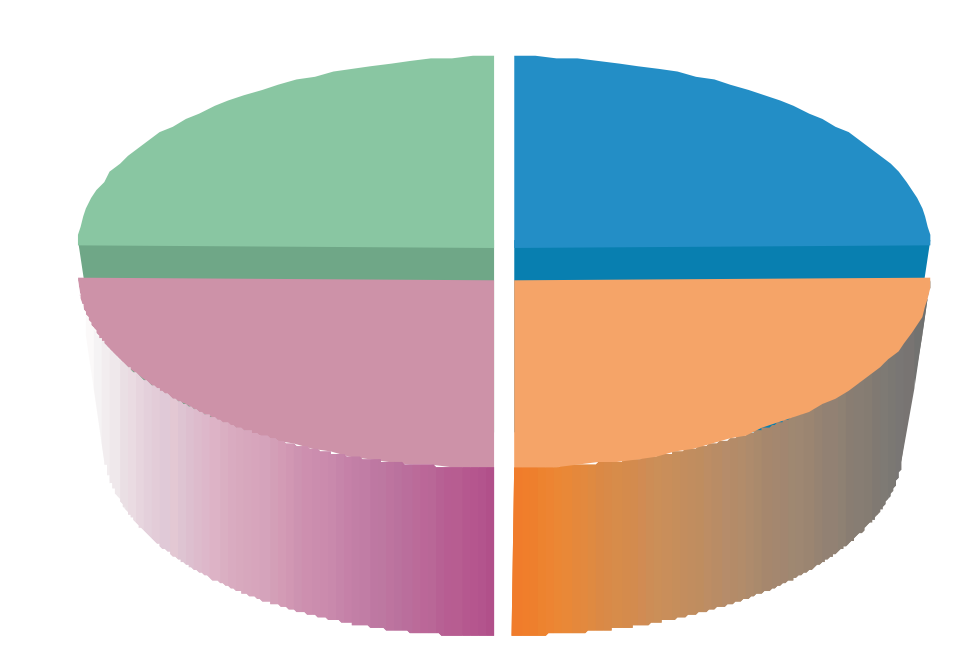

Central Server - Summary of test results
Figure 2.

System architecture.

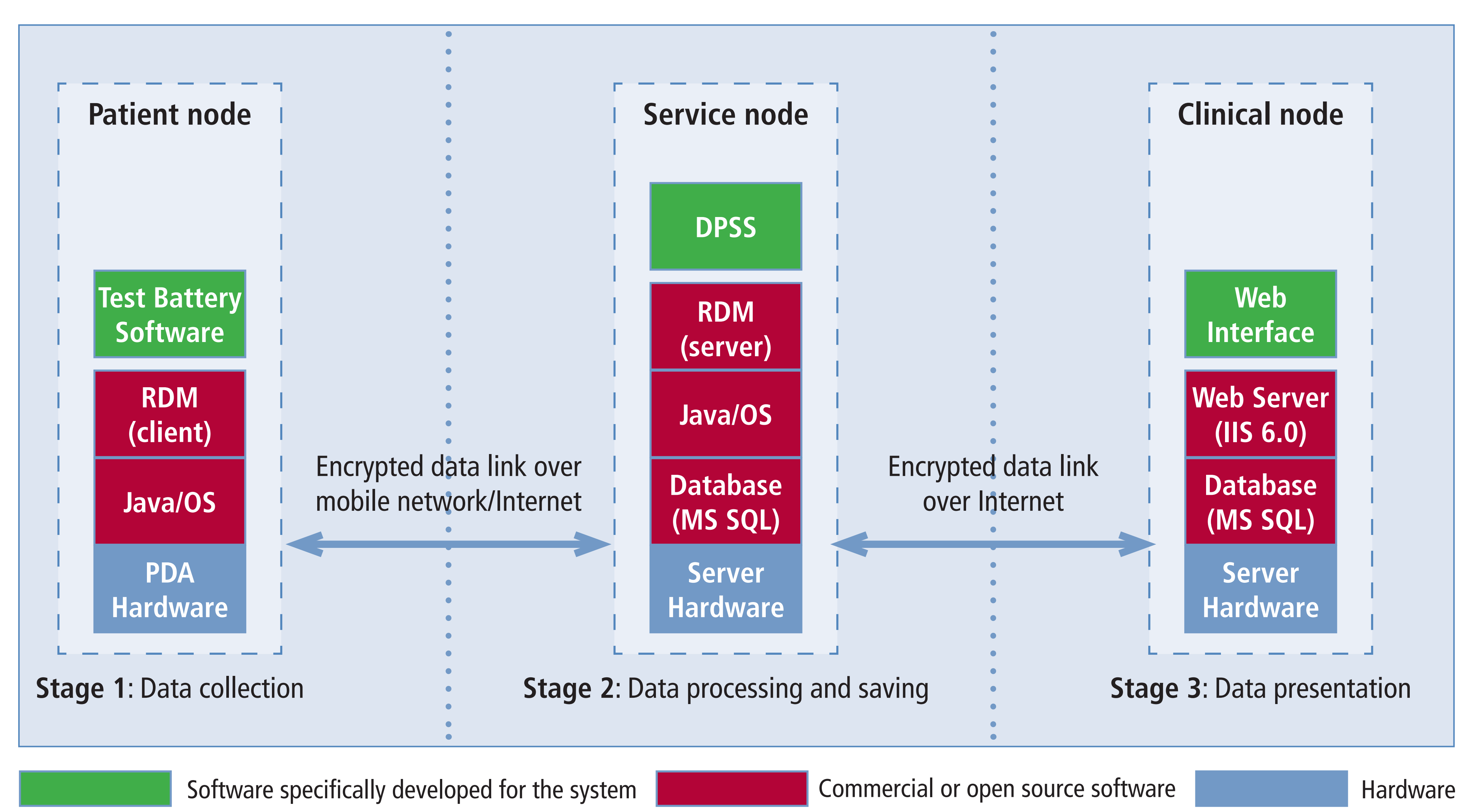

\section{Conclusions}

The system is able to summarise time series of motor test results and selfassessments during test periods and present them in a useful manner. Its main contribution is a novel and reliable way to capture and easily access symptom information from patients' home environment. Convenient access to current symptom profile, as well as symptom history, provides a basis for individualized evaluation and adjustment of treatments.

\section{Results}

The responses from nurses were positive and they are summarised in a qualitative manner as below:

- They found the web application very useful.

- They agreed with the system's assessments of the patients, which they had experience with

- They could follow complications, changes and trends within patients.

- They could compare the performance between patients.

\section{References}

1. Nyholm D, Kowalski J, Aquilonius SM, Wireless real-time electronic data capture for self-assessment of motor function and quality of life in Parkinson's disease, Mov Disord 2004; 19 (4): 446-451.

2. Westin J, Dougherty M, Nyholm D, Groth T. A home environment test battery for status assessment in patients with motor fluctuations. Comput Methods Programs Biomed. 2010; 98 (1): 27-35.

3. Westin J, Ghiamati S, Memedi M, Nyholm D, Johansson A, Dougherty M, Groth T. A new computer method for assessing drawing impairment in Parkinson's disease. J Neurosci Methods (2010), In press, doi:10.1016/j. jneumeth.2010.04.027

4. Westin J, Schiavella M, Memedi M, Nyholm D, Dougherty M, Antonini A. Validation of a home environment test battery for assessments in advanced Parkinson's Disease. Submitted to Mov Disord 2010

Figure 3.

Dimensions of the symptom state illustrated as a regular hexagon. The middle of the hexagon represents the worst score denoted by 0 and its corners represent the best score as 1 .

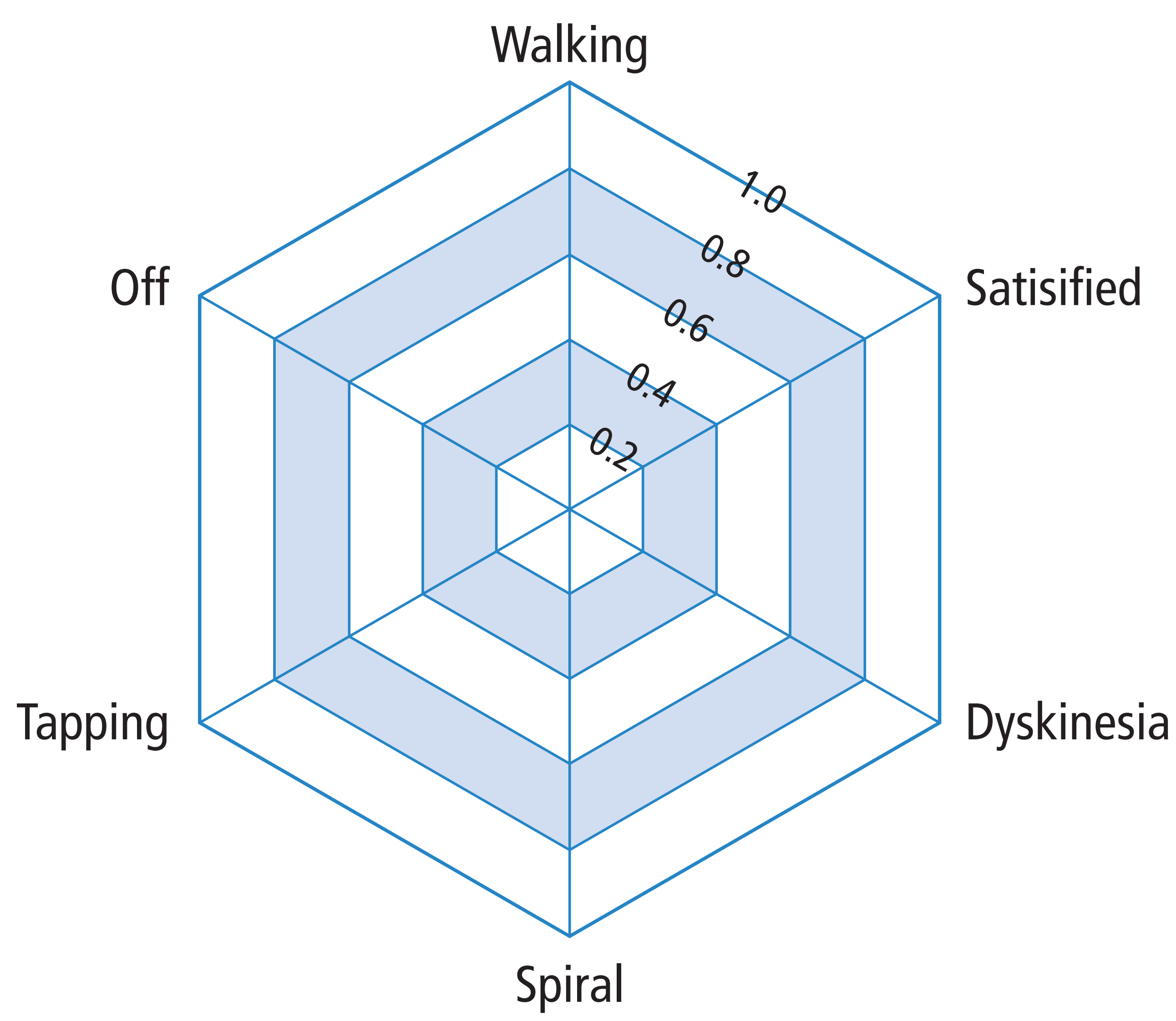

Figure 4.

Status report in the web application. Test responses for different test-battery items over a test period are shown in a time-series graph (lower left). The user can click at any designated point over the graph resulting in a popup window (upper right) showing the three spiral drawings along with their respective drawing time in seconds and the patient' self-assessment of the motor state at the time of the test occasion. Self-assessment 1 represents "a little dyskinetic" state.

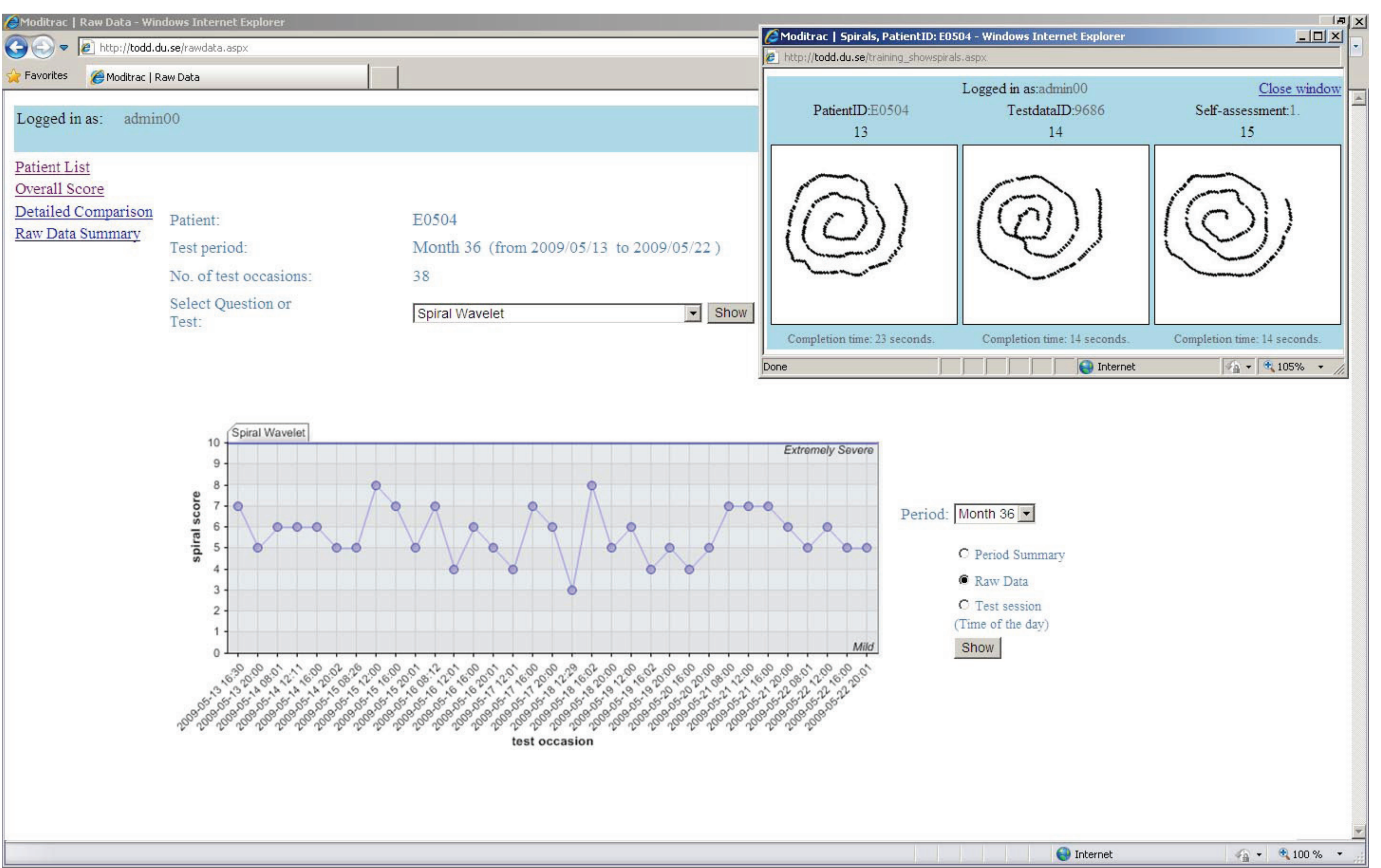

